\section{$\underset{\substack{\text { hommes } \\ \text { \& migrations }}}{ }$}

\section{Hommes \& migrations}

Revue française de référence sur les dynamiques

migratoires

$1319 \mid 2017$

Réfugiés et migrants au Liban

\title{
Pratiques marchandes et négociations identitaires
}

Le commerce ethnique dans les quartiers de Sabra et Dora (Beyrouth)

\section{Assaf Dahdah}

\section{(2) OpenEdition}

12 Journals

Édition électronique

URL : http://journals.openedition.org/hommesmigrations/3959

DOI : 10.4000/hommesmigrations.3959

ISSN : 2262-3353

Éditeur

Musée national de l'histoire de l'immigration

Édition imprimée

Date de publication : 1 octobre 2017

Pagination : 19-27

ISBN : 978-2-919040-39-1

ISSN : $1142-852 X$

Référence électronique

Assaf Dahdah, «Pratiques marchandes et négociations identitaires », Hommes \& migrations [En ligne], 1319 | 2017, mis en ligne le 01 octobre 2020, consulté le 07 janvier 2021. URL : http://

journals.openedition.org/hommesmigrations/3959; DOI : https://doi.org/10.4000/

hommesmigrations.3959 


\title{
PRATIQUES MARCHANDES ET NÉGOCIATIONS IDENTITAIRES :
}

\section{LE COMMERCE ETHNIQUE DANS LES QUARTIERS DE SABRA ET DORA (BEYROUTH)}

\author{
Par ASSAF DAHDAH, géographe, chercheur associé au CNRS (UMR 7317 LEST \\ et UMR 7303 TELEMMe).
}

\begin{abstract}
Système de contractualisation permettant aux migrants d'entrer sur le marché du travail beyrouthin par l'intermédiaire d'un Libanais, la kafala contribue à cantonner ces travailleurs dans les secteurs les plus disqualifiés. Dans deux quartiers de Beyrouth, Dora et Sabra, les migrants parviennent néanmoins à contourner l'assignation sociale et statutaire générée par ce système et à négocier leur place sur la scène marchande. Dans les deux cas, les migrants originaires d'Afrique et d'Asie affinent un dispositif commercial ethnique dont l'étude locale permet de saisir le fonctionnement et le dynamisme propres.
\end{abstract}

À l'image des pays du Golfe, le Liban s'inscrit depuis les années 1970 dans le cadre de migrations dites Sud-Sud et dans un processus de "désarabisation ${ }^{1}$ " d'une partie de la maind'œuvre étrangère. Ainsi, dans les années 2010, on estime les travailleurs non arabes à environ 300000 personnes, originaires principalement d'Éthiopie, du monde indien - Bangladesh, Sri Lanka, Népal et Inde - et des Philippines, majoritairement des femmes employées comme domestique à demeure. Cette migration est organisée selon un système d'encadrement contractuel de la mobilité et du travail dénommé : « kafala ».
La kafala ${ }^{2}$ a pour objectif de maintenir les travailleurs recrutés dans une précarité statutaire et une immobilité sociale ${ }^{3}$. Cela se traduit notamment par un cantonnement dans les secteurs les plus disqualifiés du marché du travail synonyme de faible rémunération voire d'exploitation, par un contrôle physique des migrants, en particulier des domestiques, et par une stigmatisation des personnes « altérisées ». S'il existe des formes de résistance ${ }^{4}$ au racisme et aux abus engendrés par la kafala, on peut également porter le regard sur la vie citadine des migrants qui tentent au quotidien de contourner ce régime de contraintes et d'assignation. En 
l'occurrence, il s'agit de s'intéresser spécifiquement au commerce ethnique à partir des parcours individuels, des rapports collectifs et des enjeux inhérents à l'informalité qui caractérise les secteurs dans lesquels le dispositif marchand se déploie. Pour cela, les quartiers de Dora et de Sabra ${ }^{5}$, respectivement situés à la périphérie orientale et méridionale de Beyrouth, vont être analysés ${ }^{6}$. Mais, avant d'aller plus loin et sans faire une exégèse de la littérature à ce sujet, il convient de revenir brièvement sur l'expression « commerce ethnique » et d'en préciser le sens au regard du contexte beyrouthin.

\section{Le dispositif commercial ethnique à Beyrouth}

Évoquer l'ethnicité dans les transactions commerciales signifie l'implication de négociations identitaires toujours présentes dans les relations sociales ${ }^{7}$. Emmanuel Ma Mung ${ }^{8}$ suggère de rajouter le terme de « dispositif », pri-

Il existe aujourd'hui un

grand nombre de commerces

répartis dans différents

secteurs de l'agglomération

beyrouthine qui constituent

le dispositif commercial

ethnique. L'objectif est de

s'en saisir à partir de lieux

qui ont la particularité de

concentrer un grand nombre

de ces enseignes destinées

à une clientèle asiatique et/ ou africaine. vilégiant une entrée par les acteurs, induisant une lecture davantage dynamique qui prend en compte la pluralité des relations en jeu, des statuts et des capitaux. Cette approche permet d'éviter l'écueil d'une compréhension réductrice des rapports commerciaux, et les replace dans une réalité marchande pluriethnique, multidimensionnelle et dépendant d'enjeux locaux. Ainsi, le dispositif commercial ethnique beyrouthin n'est pas homogène selon les lieux considérés, leur histoire, les acteurs et les modalités de transactions qui s'y opèrent. Tout observateur ne peut alors qu'en envisager une acception ouverte sans en négliger les particularités. Dans un contexte local donné, nous le définissons comme une activité commerciale, unique ou en complément d'une autre activité, développée par des entrepreneurs autochtones et/ ou étrangers, qui vise une ou plusieurs populations étrangères ${ }^{9}$.

Dans le contexte beyrouthin, il convient, enfin, de préciser que le fonctionnement des dispositifs commerciaux ethniques, que ce soit à Dora ou à Sabra, n'est pas régi par le confessionnalisme politique qui (dé)structure la société libanaise. Certes, il existe une hiérarchisation entre anciens et nouveaux établis, entre groupes nationaux, entre hommes et femmes, mais les activités marchandes dans les deux quartiers qui nous intéressent ici ne sont pas le lieu d'une reproduction des clivages socio-politiques habituels. Le contexte commercial tend plutôt à les transcender au profit des intérêts mercantiles des protagonistes libanais, palestiniens, indiens, bangladais, éthiopiens et philippins engagés dans ces dispositifs locaux et/ou qui tiennent les places.

Il existe aujourd'hui un grand nombre de commerces répartis dans différents secteurs de l'agglomération beyrouthine qui constituent le dispositif commercial ethnique. L'objectif est de s'en saisir à partir de lieux qui ont la particularité de concentrer un grand nombre de ces enseignes destinées à une clientèle asiatique et/ou africaine. Il ne s'agit pas d'une comparaison entre Dora et Sabra, mais d'une mise en miroir pour exposer l'hétérogénéité au sein de ce dispositif à la lumière des différents enjeux qui l'animent selon les contextes locaux.

\section{Dora, une centralité commerçante et migratoire}

Suite au génocide de 1915, des milliers d’Arméniens fuient les autorités ottomanes pour se réfugier dans

5. Voir aussi dans ce numéro l'analyse de deux interactions entre des migrants bangladais et des installés arabe sur le marché de Sabra. 6. L'analyse se fonde sur les observations et les entretiens réalisés durant la thèse de doctorat de l'auteur, soutenue en décembre 2015 sous la direction de Virginie Baby-Collin et de Michael F. Davie à Aix-Marseille Université. Elle est le résultat d'une recherche démarrée à Beyrouth en 2010, ayant abouti à la réalisation d'une centaine d'entretiens. 7. Philippe Poutignat, Jocelyne Streiff-Fenart, Théories de l'ethnicité, Paris, PUF, 2008. 8. Emmanuel Ma Mung, "Entreprise économique et appartenance ethnique ", in Revue Européenne des Migrations Internationales, vol. 12, $n^{\circ}$ 2, 1996. 9. Assaf Dahdah, " Habiter la ville sans droits. Les travailleurs migrants dans les marges de Beyrouth ", thèse de doctorat en géographie, Aix-en-Provence, Aix-Marseille Université, 2015. 
les territoires contrôlés par les troupes françaises et anglaises. Au Liban, avec l'accord de l'administration mandataire et sous la protection de la Société des Nations, cela aboutit à la construction de camps à proximité de Beyrouth et à la naturalisation des réfugiés après le traité de Lausanne (1923). Les camps vont progressivement s'urbaniser pour devenir des quartiers où peut se déployer une tradition marchande et artisanale, vecteur d'insertion pour les exilés ${ }^{10}$. Bourj Hammoud et le quartier de Dora constituent des centralités économique et communautaire pour la population arménienne qui, par la suite, les quitte progressivement pour s'installer à Beyrouth intramuros et dans la périphérie plus lointaine où l'offre immobilière s'avère plus moderne.

En raison des combats à Beyrouth à partir de 1975, Dora devient une centralité économique de substitution où se regroupent les établissements financiers et commerciaux qui ont fui les quartiers centraux ${ }^{11}$. De plus, une partie de la population arménienne déplacée y trouve refuge. Mais ce dynamisme est éphémère, puisque la cessation des hostilités autorise les établissements bancaires à retourner vers les quartiers centraux et aux habitants qui en ont les moyens de déménager de nouveau. Dora va alors connaître une période de déclin qui se traduit par la vacance de locaux commerciaux et de logements généralement petits et dégradés. Les propriétaires en recherche d'une rente sont contraints de baisser les loyers dans un quartier pourtant bien situé par rapport aux centralités beyrouthines et bien desservi par les transports collectifs. L'accessibilité et le faible niveau des loyers vont constituer les «structures d'opportunité $e^{12}$ " à l'origine de l'installation des migrants et du développement du commerce ethnique dans un quartier où la tradition marchande est solidement ancrée.

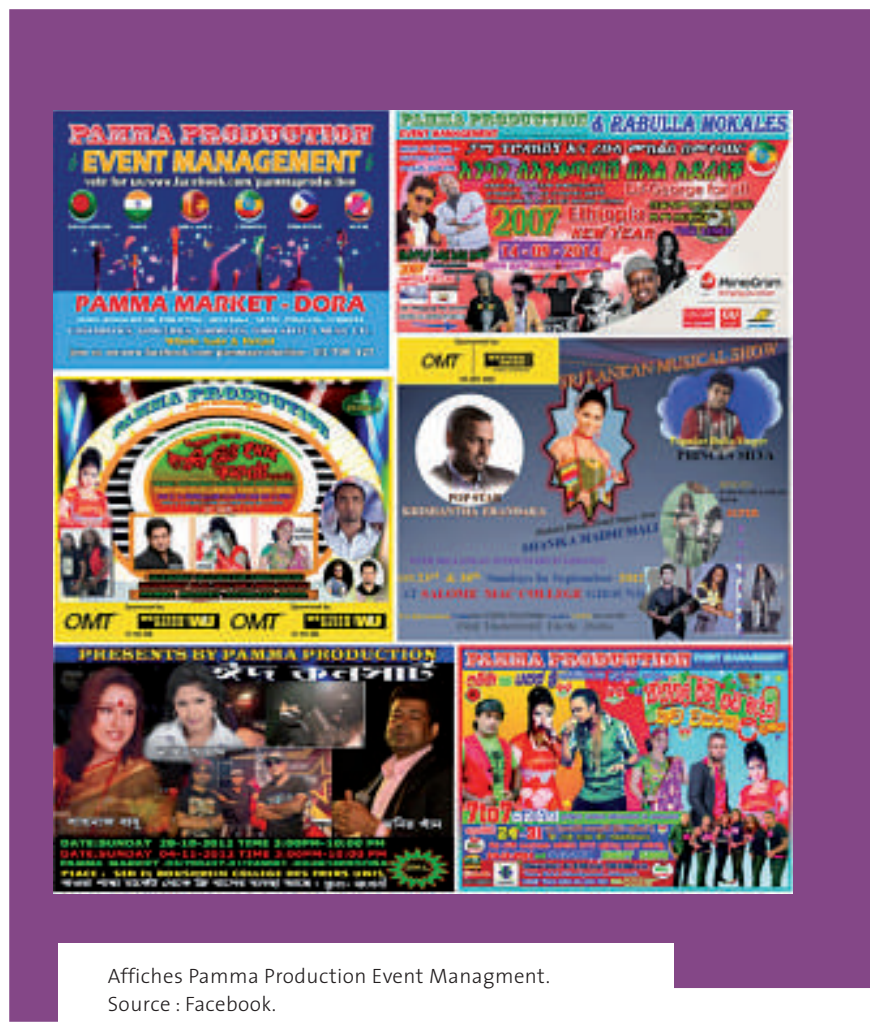

Source : Facebook.

\section{Sabra, l'internationalisation d'une marginalité urbaine}

Il est impossible de saisir les dynamiques marchandes qui caractérisent aujourd'hui ce quartier sans mettre en perspective son histoire intimement liée à celle du camp de Chatila situé à proximité, celle des Palestiniens et de leur marginalisation, et sans tenir compte de la précarité qui affecte l'ensemble des habitants. Pour autant, Sabra est également un lieu qui présente une situation cosmopolite, animée par des transactions marchandes et sociales d'une grande intensité, où s'est "greffe $e^{13}$ " un système commercial bangladais. 
Avant les guerres libanaises, le quartier de Sabra, à la périphérie de la ville, était une pinède, abritait des abattoirs et a vu se développer le camp de Chatila. Rapidement saturé et malgré l'interdiction faite par les autorités libanaises de construire dans le périmètre alloué, le camp palestinien se transforme et constitue un noyau d'urbanisation autour duquel vont être construits des logements et se développer des activités - commerces, hôpital, écoles. À l'instar de ce qui a été présenté pour Dora, les combats à Beyrouth entre 1975 et 1976 vont entraîner un déplacement des populations et des activités marchandes vers les quartiers périphériques. C'est dans ce contexte que le marché de Sabra voit le jour.

Il est difficile de retracer avec précision les événements qui vont conduire à l'avènement du dispositif commercial bangladais à Sabra. Toutefois, à travers les entretiens et les maigres statistiques disponibles, il est possible de relever trois points susceptibles d'en expliquer l'origine : tout d'abord, le rôle pionnier d'un migrant qui a démarré son activité dès 2003 ; ensuite, la croissance subite et rapide de la migration bangladaise vers le Liban à la fin des années 2000 a pu provoquer une augmentation de la demande de produits du monde indien, une aubaine pour des migrants qui tentent alors une mobilité sociale par le biais du commerce ; enfin, et malgré les tensions, Sabra reste un lieu ouvert, de coprésence et de transactions, où l'arrivée d'une nouvelle population, de nouveaux marchands, peut également signifier un gain d'argent pour ceux qui tiennent les places.

\section{Deux décennies de transformation de l'économie ethnique}

Pendant les guerres libanaises (1975-1990), le pays ne s'est jamais coupé des circulations et des échanges internationaux. Outre les trafics liés à la guerre, les migrations n'ont pas cessé : déplacements et exil des populations fuyant les combats certes, mais également immigration en provenance de Syrie, d'Égypte, du Soudan et du monde indien. Ainsi, on évoque la présence de travailleurs indiens et de domestiques originaires du Sri Lanka et des Seychelles dès la fin des années $1970^{14}$. Cependant, cette migration est freinée par le conflit et ne connaît son essor qu'à partir du début des années $1990^{15}$.

À l'aune des entretiens réalisés à Beyrouth, il semble que le développement du dispositif commercial ethnique a commencé par un double "commerce à la valise $^{16}$ » : d'un côté, les migrants se procurent des marchandises à leur retour dans leur pays d'origine et diligentent des personnes de leur entourage en circulation pour s'approvisionner régulièrement ; de l'autre, stockant leurs produits dans des valises, ils vont démarrer leur activité marchande en s'installant sur les trottoirs de Dora ou en circulant dans différents quartiers de la ville. Mais ce type de commerce va progressivement disparaître, remplacé par un système plus stable et plus sophistiqué, avec la participation de commerçants libanais.

À la fin des années 1990, Dora ne compte qu'un seul commerce indien, Pamma Indian Market, dont le gérant était initialement un ouvrier profitant de son jour de congé hebdomadaire pour vendre sur les trottoirs. Des commerçants libanais comme Atallah Est. vont à leur tour importer et proposer des produits du monde indien. Ces deux établissements, situés sur l'artère centrale "avenue Arménie », constituent ainsi des pionniers d'un dispositif qui s'est depuis enrichi.

Actuellement, une dizaine d'enseignes ont pignon sur rue tandis que les autres sont situées dans les ruelles secondaires ou dans les quartiers adjacents. Parmi elles, on constate la diversification de la clientèle visée - éthiopienne, philippine, bangladaise, soudanaise -, et des activités et des services proposés: vente de produits alimentaires et

14. Ray Jureidini, "L'échec de la protection de l'État : les domestiques étrangers au Liban ", in Revue européenne des migrations internationales, vol. 19, $n^{\circ}$ 3, 2003. 15. Selon le ministère du Travail libanais, moins de 20000 migrants non arabes travaillent au Liban en 1994. 16. Michel Péraldi, «L'esprit de bazar. Mobilités transnationales méghrébines et sociétés métropolitaines. Le comptoir démantelé ", in Michel Peraldi (dir.), Cabas et containers. Activités marchandes informelles et réseaux migrants transfrontaliers, Paris, Maisonneuve et Larose, 2001. 
agroalimentaires, salons de coiffure, restauration, import-export, prêt-à-porter, transfert d'argent, téléphonie. Nonobstant les réticences des autorités municipales et de certains habitants, les travailleurs migrants sont devenus des acteurs locaux de premier plan et le développement du dispositif a permis de redynamiser une économie moribonde. Dora est indéniablement la centralité migratoire africaine et asiatique dans l'agglomération beyrouthine.

\section{Nécessité de contourner la loi et besoin de légitimité}

Combinée à une législation discriminatoire quant aux droits des étrangers ${ }^{17}$ - restrictions quant à l'accès à la propriété privée ${ }^{18}$, au marché du travail ou à la nationalité -, la kafala et les pratiques des autorités génèrent de facto et de jure de nombreux obstacles que les migrants doivent franchir pour devenir commerçants. Subséquemment, trois alternatives s'offrent à eux : ouvrir un commerce et travailler sans autorisation; collaborer avec des Libanais qui deviennent leur prête-nom et/ou leurs employeurs plus ou moins déclarés ; se marier pour être en mesure de s'établir durablement et d'exercer une activité marchande en toute légalité.

À ma connaissance, il n'existe pas de commerce ouvert illégalement par des migrants à Dora. Même si l'informalité régit ce quartier (à l'instar de toute l'économie libanaise), les autorités municipales sanctionnent les activités trop ouvertement illégales, surtout si elles impliquent des ressortissants 
étrangers ${ }^{19}$. Quoi qu'il en soit, peu sont en mesure d'ouvrir seuls un commerce, même dans des secteurs reculés où les autorités sont moins présentes et regardantes, d'abord en raison des contraintes légales et ensuite par manque de ressources et de capital.

Partant, ils vont chercher à s'associer avec un Libanais pour louer un local, l'aménager et démarrer l'activité marchande, quand ce n'est pas un Libanais qui en est à l'initiative. Cette collaboration revêt différentes formes : un Libanais ouvre un magasin, il est présent et emploie, légalement ou non, un(e) ou plusieurs migrant(e)s ; un Libanais sert de prête-nom à une activité gérée uniquement par des migrants ; des unions entre Libanais(es) et migrant(e)s sont un moyen de contourner les obstacles administratifs et d'acquérir de la légitimité, pour l'un auprès d'une clientèle méfiante et méconnue, pour l'autre dans un contexte local où la mobilité sociale d'un travailleur migrant est généralement mal perçue, d'autant plus si c'est une femme sensée être au Liban pour travailler comme agent d'entretien ou domestique.

Les unions - concubinage, mariage - permettent donc de dépasser ces différentes contraintes. Dans la majorité des cas, elles contribuent pour le conjoint libanais à combler la méconnaissance des pratiques, des codes et de la langue, et son manque de reconnaissance parmi le groupe national ciblé, toujours essentiellement féminin ${ }^{20}$. Il profite de sa conjointe pour se constituer une clientèle, laissant dire à des commerçants interrogés que certaines unions sont uniquement intéressées et de circonstances ${ }^{21}$. En somme, les relations de concubinage ou maritales visent à s'arranger avec les contraintes induites par le contexte légal et migratoire, et à optimiser le "capital social ethnique $e^{22}$ " indispensable à la réussite d'une entreprise commerciale à Dora.

\section{Fragilités et verticalisation du dispositif}

Le dispositif connaît plusieurs réussites économiques participant à structurer et à transformer le tissu commercial du quartier. Elles sont toutefois le pendant d'une réalité plus contrastée qui implique également une plus grande instabilité. Celle-ci résulte, tout d'abord, de la crise économique de 2008 qui a fortement touché le Liban. C'est, ensuite, la concurrence que se livrent les multiples protagonistes du marché dont les activités sont peu ou prou similaires. Enfin, conséquence indirecte de la guerre en Syrie, les autorités ont décidé de mener une politique sécuritaire qui s'est traduite par des interventions policières et par des arrestations répétées de migrants résidant illégalement au Liban. Ces trois facteurs ont inévitablement entraîné un redéploiement du dispositif commercial, dont une partie des enseignes a fermé.

Dans leur majorité, les acteurs historiquement installés ont su résister et s'adapter à cette situation. Ils ont renforcé leur position en devenant à la fois détaillants et grossistes. Autrement dit, pour limiter les frais de transport, augmenter leurs marges et se distinguer des autres commerçants, ils ont fait le choix d'investir pour importer par containers de grandes quantités de marchandises, essentiellement depuis Dubaï. Des investissements que les enseignes plus modestes ne sont pas en capacité de réaliser et dont l'activité finit par péricliter.

Le secteur de l'événementiel a connu une évolution comparable. Il a émergé à la fin des années 2000 lorsque Charly Cargo, un commerçant libanais, a décidé d'organiser des concerts de vedettes éthiopiennes. Par la suite, plusieurs commerçants motivés par sa réussite ont décidé de l'imiter, multipliant les dates pour attirer une clientèle pourtant

19. Plusieurs lieux ont été fermés ces dernières années suite à des présomptions et des accusations de prostitution.

20. Selon le ministère du Travail, conséquence d'une immigration organisée presque exclusivement autour de la domesticité féminine, plus de $90 \%$ des migrants non arabes au Liban sont des femmes. 21. Au Liban, seuls les hommes peuvent transmettre leur nationalité à leur épouse et à leurs enfants. Par conséquent, seul un mariage peut sécuriser le statut des femmes étrangères. Le concubinage maintient donc de nombreuses femmes dans l'incertitude et l'instabilité puisqu'elles restent sans droits. Les unions entre Libanaise et migrant existent également, bien que plus rares, et permettent aux hommes d'obtenir un permis de résidence renouvelable tous les cing ans. 22. Alexander Nicholls, "Capital social ethnique et entrepreneuriat. Le cas des commerçants chinois de Paris, Bruxelles et Montréal ", in Sociologie, vol. 3, $n^{\circ} 4,2013$. 
peu fortunée. Après plusieurs échecs, les grands événements se sont arrêtés. C'est alors que le gérant de Pamma Indian Market a créé une filiale nommée Pamma Production Event Managment, aujourd'hui en position de quasi monopole pour l'organisation des concerts à destination des ressortissants du monde indien et d'Éthiopie.

Somme toute, en l'espace de deux décennies, Dora est devenu une place commerciale incontournable pour tout migrant et Libanais qui souhaite s'insérer dans les réseaux marchands. Le dispositif commercial ethnique fait partie intégrante de son paysage, anime désormais les transactions et les interactions, et structure ce quartier périphérique de la capitale libanaise.

\section{Prendre place à Sabra et s'y maintenir}

Contrairement à Dora, le dispositif de Sabra est caractérisé par son homogénéité et sa concentration. En effet, celui-ci est exclusivement bangladais et se limite à un périmètre réduit du quartier. Toutefois, cette place marchande animée, dense, est complexe pour tout observateur qui souhaite en saisir le fonctionnement. Initié au début des années 2000 avec l'installation du premier commerçant bangladais, le système commercial s'est depuis développé avec l'arrivée de vendeurs de rue et l'ouverture de nouvelles enseignes. Mais il connaît 
également une forte instabilité liée à l'informalité des activités, et à des rapports de domination d'une grande intensité révélatrice de la « lutte des places ${ }^{23}$ » entre les divers acteurs qui habitent ce secteur précarisé.

Le premier marchand bangladais présent dans le quartier serait Mounir ${ }^{24}$, un homme d'une quarantaine d'années arrivé au Liban au début des années 1990 dans le cadre d'une migration contractualisée. À l'instar du créateur de Pamma Market, Mounir profite de ses jours de congés pour circuler dans les rues de Beyrouth, vendant les produits que lui et ses amis ramènent du Bengladesh lors de leurs voyages. Résidant à proximité de Sabra, il fréquente régulièrement ce marché populaire où il se poste également le dimanche avec sa marchandise. En 2003, il décide de changer de stratégie et de se fixer dans le quartier à la faveur d'une

Contrairement à Dora,

le dispositif de Sabra

est caractérisé par son

homogénéité et sa

concentration. En effet,

celui-ci est exclusivement

bangladais et se limite à un périmètre réduit du quartier. place nouvellement vacante ; un parfumeur syrien a décidé de rentrer en Syrie, permettant à Mounir de louer un modeste immobilier - une armoire métallique scellée à même le trottoir. Jusqu'en 2009, Mounir est le seul marchand bangladais de Sabra, une activité qu'il exerce désormais à plein temps et une situation de monopole qui lui permet de réaliser un chiffre d'affaire conséquent - presque 1000 dollars par semaine. La situation change à partir de 2009 avec l'arrivé de Sultan, comme lui un migrant contractuel, commis de cuisine qui complète ses revenus avec la vente de produits bangladais. Puis, à partir de 2011, une dizaine de vendeurs bangladais s'installe sur les trottoirs de Sabra avec cartons, cagettes et tables en plastique, et parasols. Puis de nouvelles enseignes apparaissent. Ce que Mounir considère initialement comme une aubaine, c'est-à-dire la venue de marchands qui rendent ce marché attractif pour de nouveaux clients, devient finalement une concurrence qu'il ne parvient pas à contrer. Pour se distinguer, il ouvre un restaurant dans le groupement palestinien ${ }^{25}$ auquel son activité est adossée ; le dimanche, journée d'affluence, le lieu est bondé. Ainsi, en quelques années, le marché de Sabra est devenu le principal rendez-vous de la population bangladaise de l'agglomération beyrouthine. Mais, à la différence de Dora, à Sabra seuls les migrants sont les entrepreneurs de cette économie ; les établis palestiniens et libanais contrôlent quand à eux les places qu'ils monnaient chèrement.

\section{Le rôle des placeurs dans un quartier dense et disputé}

Ceux que l'on peut nommer les «placeurs de Sabra » sont des figures locales qui, du fait de leur position, sont en mesure de réglementer l'accès au marché et de taxer les vendeurs de rue, non sans tensions entre placeurs et marchands, et entre placeurs euxmêmes dans un contexte de rivalités. Ainsi, les responsables des groupements palestiniens mitoyens via leurs cailds, les chauffeurs de taxi et les commerçants exigent-ils des Bangladais une somme hebdomadaire ou mensuelle en fonction de la taille de l'emplacement et de sa situation. Les commerçants s'arrogent le droit de louer le trottoir situé devant leur pas-de-porte à des vendeurs qui souhaitent s'y installer. Ces derniers doivent également s'affranchir d'une somme - de 5000 LL à 15000 LL soit entre 3 euros et 10 euros environ - que viennent prélever les autres placeurs patentés. Une forme de racket auquel ils ne peuvent résister étant donnés les rapports de force qui animent le marché.

Cette contrainte qui pèse sur les Bangladais n'empêche pas le développement du dispositif stricto sensu, mais elle en conditionne le fonctionnement qui est ainsi soumis à une hiérarchisation et à une instabilité auxquelles ils ne sont pas en mesure de déroger. Les placeurs rappellent en permanence aux migrants combien leur place, en tant que nouveaux venus sans pouvoir, est précaire et qu'ils peuvent à 
tout moment les en priver. Ces interactions peuvent se dérouler sur le ton de l'ironie, mais également être synonyme de brutalité et d'humiliation. Ils leur refusent en outre toute forme d'activité qui pourrait les placer en concurrence avec celle des établis. Si certains bouchers emploient des Bangladais et possèdent une enseigne écrite en bengali pour attirer le chaland, en revanche aucun migrant n'exerce comme boucher, profession considérée comme une position réservée aux Palestiniens et aux Libanais. Le marché bangladais de Sabra n'a eu de cesse d'évoluer depuis ses débuts. Les pionniers Mounir et Sultan ont revendu leur fond de commerce et sont repartis au Bangladesh. Dans le même temps, de nouveaux commerçants ont pignon sur rue, presque tous situés dans une même ruelle du quartier. Le dispositif paraît donc encore plus concentré qu'auparavant, mais il reste instable. Un processus qui s'est amplifié avec le déplacement des vendeurs de rue suite à l'intervention des forces de police en 2014 qui a entraîné le départ temporaire ou définitif de nombreux biffins bangladais.

L'informalité qui caractérise le marché de Sabra constitue à la fois une opportunité et une contrainte. Une opportunité car elle offre la possibilité aux migrants de contourner les restrictions imposées par le système de la kafala et d'initier, sous la protection/le contrôle des placeurs, une activité commerciale. Une contrainte car elle signifie par ailleurs une vulnérabilité et une grande instabilité dans un secteur où les rapports de domination, conditionnés par la hiérarchisation des identités, restent omniprésents.

\section{Conclusion}

Comme on a pu le voir à travers le contexte beyrouthin, les migrations internationales ne peuvent être appréhendées sans tenir compte des échelles locales et des réalités urbaines dans lesquelles les migrants s'inscrivent. Mais elles ne sont pas non plus déconnectées des réglementations nationales qui encadrent les mobilités et hiérarchisent les identités.

Le dispositif commercial ethnique représente un vecteur de mobilité sociale et de reconnaissance pour des personnes " altérisées ", vouées à n'être que des employés interchangeables et sous-payés. L'identité des migrants, protagonistes étrangers aux tensions locales et nationales, leur permet de se jouer des catégorisations et des crispations héritées des conflits moyen-orientaux pour se faire une place. Par leur présence, par la diversité des langues et des pratiques marchandes, ils contribuent à internationaliser les lieux dans lesquels ils s'inscrivent. Cependant, cette identité constitue en même temps une étiquette à laquelle ils sont systématiquement renvoyés afin de leur rappeler qu'ils restent des individus dominés et à la légitimité toujours contestée. Ce faisant, comme le soulignent Alain Morice et Swanie Potot, «il convient d'écarter toute vision idyllique des rapports sociaux, souvent très durs, qui Le marché bangladais de Sabra n'a eu de cesse d'évoluer depuis ses débuts. Les pionniers Mounir et Sultan ont revendu leur fond de commerce et sont repartis au Bangladesh. Dans le même temps, de nouveaux commerçants ont pignon sur rue, presque tous situés dans une même ruelle du quartier. traversent le monde de l'immigration, et qui sont le reflet accentué de ce que, globalement, les pays d'accueil lui font subir ${ }^{26}$ ".

Fort de ce constat, on peut dire que le commerce ethnique à Sabra et Dora ne représente pas véritablement une rupture avec la migration contractualisée et ses inégalités instituées. S'il est vrai que les migrants profitent des failles du système, de l'informalité qui caractérise ces secteurs périphériques pour contourner une immobilité sociale et spatiale imposée, les parcours individuels et les relations sociales rappellent combien la précarité statutaire et l'assignation identitaire des travailleurs africains et asiatiques au Liban restent prégnantes. 